\title{
A METHOD OF FINDING IMAGE SIMILAR PATCHES BASED ON GRADIENT-COVARIANCE SIMILARITY
}

\author{
Yesen Sun
}

Department of Mathematics, Jinan University, Guangzhou 510632, P. R. China

\begin{abstract}
In this paper, we propose a new method of searching for image similar patches after image blocking processing, which combines the gradient information of image and the covariance similarity measure. Gradients convey important visual information and are crucial to scene understanding, covariance similarity measure used to measure the similarity of two variables is very useful, which can capture the image structure and the change of the contrast more effectively in together. Compared with covariance method, the projection method, and face recognition method respectively finding similar patches, a large number of experimental results show that gradient-covariance method is much better than other methods.
\end{abstract}

Keywords: image blocking, similarity measure, image gradient, similar patch.

${ }^{*}$ Corresponding author.

E-mail address: 1096268556@qq.com (Yesen Sun).

Copyright ( 2015 Scientific Advances Publishers

2010 Mathematics Subject Classification: 68T10, 68U10.

Submitted by Jianqiang Gao.

Received September 31, 2015 


\section{Introduction}

In image processing, image blocking technology has played an important role, and widely applied to image denoising, image restoration, image enhancement and other fields. After blocking, the influence of the close distance of the pixel is larger than the far distance, so block processing can better deal with each pixel, and get more details. The similar patch dictionary (a collection of similar patches) based on image blocking played the vital role in image restoration, image denoising, image enhancement, image feature extraction, image quality evaluation, image assessment and so on. Therefore, the similarity measurement methods are also key to find the similar patches. The most widely method searching for similar patch is the Euclidean distance, as observed in [1][4], the Euclidean distance metric is used to find similar figure of target image. A weighted Bhattacharya distance measure has been proposed in [5] to find similar patches. Note that other distance metrics also exit that account for geometrical transformations [6]. Ning et al. [7] present feature similarity and covariance matrix similarity, and raise minimized feature similarity, it can effectively apply the covariance matrix algorithm to automatic target detection with high detection rate and low false alarm rate. PD (programming dynamic) matching is a method to calculate the distance of two one-dimensional vector matching, Liu et al. [8] propose a feature matrix similarity measurement method based on PD matching. Huang and Yang [9] apply the principal componment analysis (PCA) algorithm to face recognition. It can extract the most important features of the face image, and then use the distance measurement to improve the accuracy of the recognition. In this article, we apply the second screening on the basis of looking for similar patch in European measures, and compare the three different methods of finding similar patches: projection, the face recognition method. Then, we put forward a new gradient-covariance measure method combining the gradient similarity and covariance measure. Finally, a great deal of 
experimental data approve that the method is feasible and effective, and show this method is better than the projection method, face recognition method, and the covariance method in the wrong rate searching for similar patches.

\section{Theoretical Basis}

There are many kinds of similarity measures in image, but the wide application and the relative maturity of the similarity measure can be classified into two categories: distance measurement and relative measurement. The distance function refers to that each sample is regarded as a point in the high dimensional space, and then uses some distance to represent the similarity between the sample points. Near sample points are similar and the distance between the sample points is different. There are some commonly used distances, for example, Euclidean distance, Minkowski distance, Manhattan distance, and Chebyshev distance. The relevant measure is pointed to determine the similarity between the target and the sample with any metric, it is usually defined as a cost function or distance function. The classical correlation measurement includes correlation function, covariance function, and cosine functions etc.

Assume $I$ a gray image that the size is $M \times N$, for the most part the picture is relatively large, the image processing not only wastes time but also affects the complexity, so we chose to use image blocking processing approach. We divided $I$ into $n \times n$ patches $\left\{A_{i}, i=1,2, \cdots\right\}$ of equal size,

then pick up a patch $A_{T}$ from the sub-patches $\left\{A_{i}, i=1,2, \cdots\right\}$ as target, the rest is called the candidate patches $\left\{A_{i}\right\}$. Similar dictionary is defined as the set of part of candidate patches, which is similar to target patch. 
For example, in the process of image restoration, the target image with missing elements can be repaired. First of all, the image $I$ is divided into patches $\left\{A_{i}, i=1,2, \cdots\right\}$, then we can select the target patch $A_{T}$ with missing elements and similar patches of $A_{T}$, and use similar patches of $A_{T}$ to repair the missing target patch.

\subsection{Several common methods of searching for similar patches}

(1) Euclidean metric method

The most common distance representation method between two points or more points, also known as the Euclidean metric. In the Euclidean space, the distance between points $x=\left(x_{1}, \cdots, x_{n}\right)$ and $y=\left(y_{1}, \cdots, y_{n}\right)$ in the $n$-dimension is defined as

$$
d(x, y):=\sqrt{\left(x_{1}-y_{1}\right)^{2}+\left(x_{2}-y_{2}\right)^{2}+\cdots+\left(x_{n}-y_{n}\right)^{2}}=\sqrt{\sum_{i=1}^{n}\left(x_{i}-y_{i}\right)^{2}} .
$$

Then the Euclidean distance between the target patch $A_{T}$ and the candidate patches $\left\{A_{i}\right\}$ is

$$
d_{i}=\sqrt{\sum_{j=1}^{n} \sum_{k=1}^{n}\left(x_{k j}-y_{k j}\right)^{2}}
$$

where $x_{k j}$ and $y_{k j}$ are the any one pixel of the $A_{T}, A_{i}$, respectively. $d_{i}$ is the Euclidean distance of the $i$-th patch $A_{i}$ and target patch $A_{T}$. The smaller the measure values, the more similar.

(2) Covariance similarity measures method

Covariance is used to measure the overall error of two variables in probability theory and statistics. And variance is a special case of covariance, i.e., two variables are the same. Covariance similarity measures method is denoted as COV. The covariance of two real valued random variables whose expected value is $u=E[X]$ and $v=E[Y]$, respectively, is defined as 


$$
\operatorname{cov}(X, Y)=E((X-E[X])(Y-E[Y]))
$$

Assume the pixel matrixes of the target patch and the candidate patches, respectively, correspond to $A_{T}$ and $\left\{A_{1}, A_{2}, \cdots, A_{N}, \cdots\right\}$, so the total elements of each matrix can be arranged by a column, and the elements of the matrix $A_{T}$ are arranged $b$ by a column. Similarly, the elements of each matrix in the candidate patch can be arranged in a column, denoted as $\left\{b_{1}, b_{2}, \cdots, b_{N}, \cdots\right\}$, so that it can be a large matrix after putting $b$ into $\left\{b_{1}, b_{2}, \cdots, b_{N}, \cdots\right\}$, denoted as $U:\left\{b, b_{1}, b_{2}, \cdots, b_{N}, \cdots\right\}$.

In this case, the similarity between the patch and the patch will become the similarity between the column and the column. The covariance is a measure of the correlation of the two random variables, and then the covariance measure is usually selected to use. So, the covariance function $f\left(b_{i}, b\right)$ between the each of $\left\{b_{1}, b_{2}, \cdots, b_{N}, \cdots\right\}$ and $b$ is defined as

$$
f\left(b_{i}, b\right)=E\left(b_{i}-E\left[b_{i}\right]\right)(b-E[b])
$$

According to the sort of covariance size from large to small, we select a threshold and the corresponding patches whose metric values are higher than the threshold value to achieve screening.

(3) Projection method

Using projection method finds the similar patches, which projects the image feature to a specific space, forming a feature space, and then comparing the characteristics to go to screening. This method is denoted as PRO. Specifically, after the image is divided into patches, each patch is processed according to a matrix, then the feature vector of the target patch $A_{T}$ is first obtained, then each patch projects onto the set of feature vectors $v$ as

$$
\left\{A_{1} v, A_{2} v, \cdots, A_{N} v, \cdots\right\}
$$


To calculate the distance between $\left\{A_{1} v, A_{2} v, \cdots, A_{N} v, \cdots\right\}$ and the projection $A_{T} v$ that target patch projects the feature space, we select a threshold and the corresponding candidate patches being less than the threshold to achieve screening.

(4) Using the ideas of face recognition

Combining with the idea of face recognition, covariance matrix can simultaneously show the correlation among different dimensions and the variance of each dimension. The main features of the image are extracted from the covariance matrix. And features are compared to find the similar images. This method is denoted as FRG. Specifically, this method can be divided into the following steps:

(1) Calculate the covariance matrix $C$ of the target patch $A_{T}$.

(2) Calculate eigenvalues and eigenvectors of the covariance matrix $C$. The feature values are selected from large to small, and the feature vectors are extracted as $\left\{v_{1}, v_{2}, \cdots, v_{n}, \cdots\right\}$. These feature vector $\left\{v_{1}, v_{2}, \cdots, v_{n}, \cdots\right\}$ constitute the space of feature $V$.

(3) The test image $\left\{A_{i}\right\}$ is projected onto the feature vector $V$ space to obtain the corresponding projection vector $\left\{A_{i} V\right\}$, and then same candidate image should also obtain the corresponding projection vector $A_{T} V$.

(4) Calculate the distance between the projection of the candidate patches and the projection of the target patch and sort it, selecting a threshold. Then, the corresponding candidate patch being less than the threshold should be selected as similar dictionary.

\section{Thought of Adding Gradient on the Traditional Covariance}

According to the characteristics of image, we can know that gradient can better reflect the structure characteristics of the image. Hence, considering the covariance method with the thought of gradient, we call 
it gradient-covariance method, denoted as GRA-COV. Let $f_{1}(p, q)$ denotes the covariance function between image patch $p$ and patch $q$. Let $f_{2}(p, q)$ denotes the square root function of the sum of the squares of gradient difference. Then similarity measurement between the patch and patch can be defined as follow:

$$
d= \begin{cases}f_{1} & f_{2}=0 ; \\ \frac{f_{1}}{f_{2}} & f_{2} \neq 0,\end{cases}
$$

where the covariance function $f_{1}(p, q)$ is given by

$$
f_{1}(p, q)=E(p-E[p])(q-E[q])
$$

the define of $f_{2}(p, q)$ is

$$
f_{2}(p, q)=\sqrt{\left(G_{x}(p)-G_{x}(q)\right)^{2}+\left(G_{y}(p)-G_{y}(q)\right)^{2}}
$$

where $G$ is the gradient of the image, $E$ is the mean value. When selecting the similarity patch of the target patch, we calculate the covariance similarity metric of target patch with each candidate patch, then select a threshold value, and select the corresponding patches that it metric values is higher than the threshold value as similar dictionary. 


\section{Experimental Analysis}

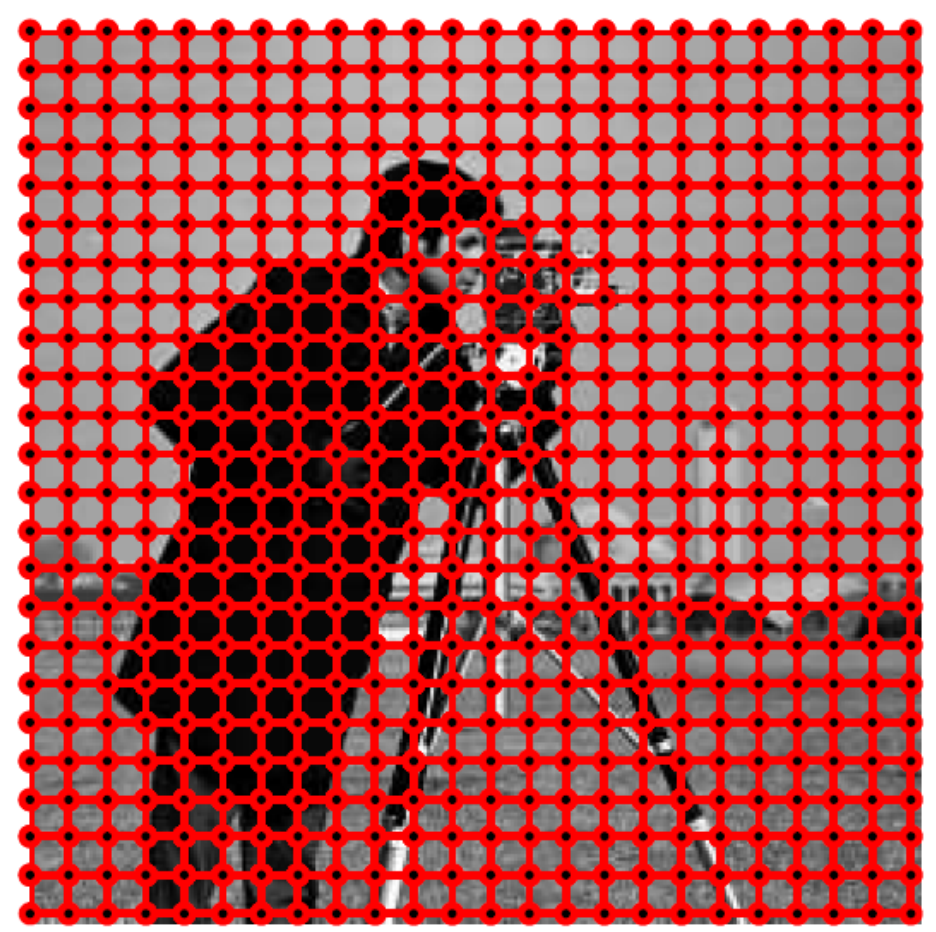

cameraman

Figure 1. The classical image cameraman.

Let us use Figure 1 as experimental material. Firstly, the image is divided into patches, the patch size is defined as $11 \times 11$, and we select the 61 lines, 126 columns of the image as the center of target patch. Then we use the Euclidean distance method to do the first screening, and choose $60,80,100,200,300$, and 400 as the sample. Secondly, then we use COV, PRO, FRG, and GRA-COV to select the patches that is similar to the target patch for the second time, and the threshold value is set to mean value of measures. The results are as follows: 


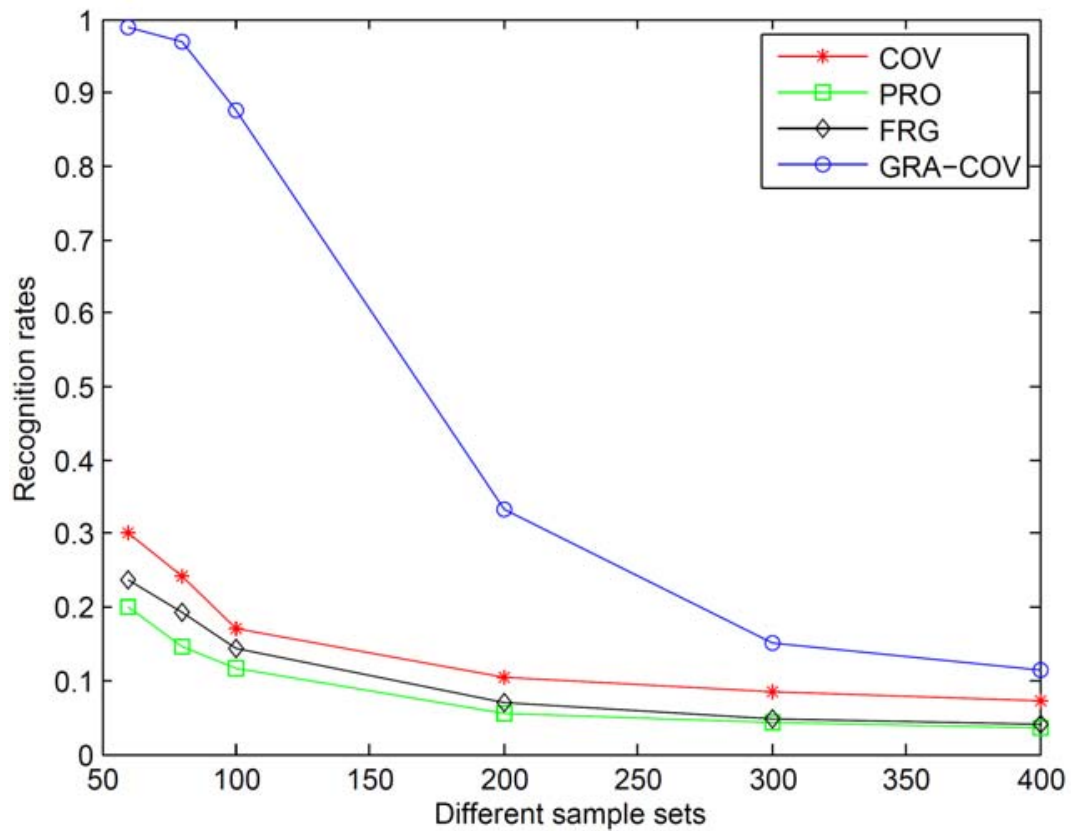

Figure 2. The recognition rate of COV, PRO, FRG, and GRA-COV in different samples.

It can be seen from Figure 2 that the recognition rate of the covariance method, projection method and face recognition method is less different in selecting similar patches. But the recognition rate of covariance gradient method is the best among the several methods, especially in less sample set.

\section{Conclusion Analysis}

In this paper, a new method for searching for the similar patches of target patch is proposed. It is a gradient-covariance method based on image structure characteristics, then comparing it with covariance method, projection method and face recognition method. By empirical analysis, we can get the following conclusions: (1) Compared with the other three methods, the recognition rate of the gradient-covariance method in finding a similar patch is the highest. (2) The recognition rate of gradient-covariance method is much higher than the other methods, 
especially in case of less sample set. In image similarity retrieval, selecting high similar patches is important in image processing, especially in image feature extraction, image restoration, image denoising and so on. Using similar patches to restore missing areas or remove noise, and it can greatly reduce the retrieval time in image retrieval. But the experiment is built on the basis of the feature that target patch is obvious, the experimental results are distinguished by eyes, there are small errors in this method. However, I believe this paper's thought searching for similar patches, as a new processing direction, will find more applications in the future.

\section{References}

[1] A. Criminisi, P. Perez and K. Toyama, Region filling and object removal by examplar-based image inpainting, IEEE Trans. Image Process. 13(9) (2004), 1200-1212.

[2] A. Efros and T. Leung, Texture synthesis by non-parametric sampling, In Proc. Int. Conf. Computer Vision, pages 1033-1038, Kerkyra, Greece, 1999.

[3] Z. Xu and J. Sun, Image inpainting by patch propagation using patch sparsity, IEEE Trans. Image Process. 19(5) (2010), 1153-1165.

[4] O. Le Meur, J. Gautier and C. Guillemot, Examplar-based inpainting based on local geometry, in Proc. 18th IEEE ICIP (2011), 3462-3465.

[5] A. Bugeau, M. Bertalmio, V. Caselles and G. Sapiro, Real-time texture synthesis by patch-based sampling, ACM Trans. Graph. 2(3) (2001), 127-150.

[6] Y. Zhang, J. Xiao and M. Shah, Region completion in a single image, in EUROGRAPHICS, 2004.

[7] Z. L. Ning, H. Q. Wang and Z. Zhang, An automatic object detection method based on covariance matrix, 27(3) (2010), 370-375.

[8] Y. H. Liu, F. Wang and X. D. Liu, A feature matrix similarity measure method and its application to image retrieval, J. Pattern Recognition and Artificial Intelligence 19(4) (2006), 497-502.

[9] H. S. Huang and A. Q. Yang, Face recognition based on PCA algorithm, Electronic Science and Technology 28(8) (2015), 98-101.

[10] A. Liu, W. Lin and M. Narwaria, Image quality assessment based on gradient similarity, Image Processing, IEEE Transactions 21(4) (2012), 1500-1512. 